\title{
ELASTIC POTENTIALS AND THE STRUCTURE OF INELASTIC CONSTITUTIVE LAWS*
}

\author{
R. HILL† AND J. R. RICE $\ddagger$
}

\begin{abstract}
The existence of a work potential, governing material response when elastic behavior occurs, is shown to provide a concise framework for the structure of inelastic constitutive laws. The discussion includes the identification of differential invariants in conjugate stress and strain variables, an examination and reformulation of postulates leading to "normality rules", and a study of the transmission of constitutive properties from subelements to overall behavior of heterogeneous systems. Also, internal variable representations of inelasticity are examined within this framework.
\end{abstract}

1. Introduction. We consider elastic/plastic materials of a general kind, either time-independent or time-dependent, which have the property that their purely elastic response admits a work potential. We show how this property can form the basis of a concise framework for the structure of their inelastic constitutive laws. The formalism is well suited to discussing macroscopic properties of materials which are heterogeneous on the microscale.

This approach is motivated by the work of Kestin and Rice [1] and Rice [2] on internal state-variable models for plasticity. The postulated existence of thermodynamic potentials was there shown to lead to an explicit relation between inelastic strain-increments and changes in the variables. A class of kinetic rate laws for the variables was thereby identified which implies a normality structure in the macroscopic constitutive equations. By contrast we proceed here without specific reference to internal variables, though we subsequently introduce them as one possible way to represent the history dependence of inelastic deformation.

We show further how invariance theorems in the general class established by Hill [3] can be derived concisely in the event that elastic potentials exist. The invariance in question refers not only to the whole family of conjugate measures of stress and strain, but also to the transmissibility of constitutive properties between microscopic and macroscopic levels.

\section{Inelastic materials and elastic potentials.}

2.1. Basic notions. Let e denote any strain tensor, objective and symmetric, that measures deformation from an arbitrary reference state. Let $\mathbf{t}$ be the symmetric conjugate stress such that $\mathbf{t} d \mathbf{e}$ is the work per unit volume of the adopted reference state in any virtual deformation $d \mathbf{e}$.

We consider inelastic materials that exhibit, under certain loadings, a purely elastic response at any stage of deformation. Such response may, for example, be elicited only by stress variations whose directions and magnitudes fall in a certain range, or perhaps only as a limit when the variations are sufficiently rapid. By

* Received by the editors March 2, 1973.

† Department of Applied Mathematics and Theoretical Physics, Unıversity of Cambridge, Cambridge, England.

$\ddagger$ Division of Engineering, Brown University, Providence, Rhode Island 02912. This work was supported in part by an NSF Senior Postdoctoral Fellowship and by the U.S. Atomic Energy Commission. 
elastic we mean, in the first instance, that the (t, e) relation is one-to-one, though it depends on the prior inelastic deformation. This same relation, when evaluated at the current strain, gives the current stress whether subsequent deformations are elastic or not.

We can therefore write

$$
\mathbf{t}=\mathbf{t}(\mathbf{e}, H)
$$

where the notation is intended to convey that $\mathbf{t}$ is a direct function of $\mathbf{e}$ and a functional of the prior history of inelastic deformation (denoted symbolically by $H$ ). For each $H$ the function is defined in some domain of strain space which necessarily includes the current state, though possibly as a boundary point. The variation of $\mathbf{t}(\mathbf{e}, H)$ over the domain at a particular $H$ is then descriptive of the elastic response at that $H$.

Our basic assumption is that the response is always of Green type, in the generalized sense that a work potential

$$
\phi=\phi(\mathbf{e}, H)
$$

exists at each $H$ and within the associated e domain. Then, for any such strain variation $\delta \mathbf{e}$ at fixed $H$, which is to say during purely elastic deformation,

$$
\mathbf{t} \delta \mathbf{e}=\delta \phi(\mathbf{e}, H) .
$$

Hence

$$
\mathbf{t}=\frac{\partial \phi(\mathbf{e}, H)}{\partial \mathbf{e}}
$$

when $\phi$ is symmetrized in the components of e. Subsequent developments follow directly from the validity of this equation throughout any program of inelastic deformation. It is to be understood that $\phi$ is taken at each stage as the potential appropriate to the accumulated history $H$ of inelastic deformation.

Dually, in terms of the complementary potential

$$
\psi=\psi(\mathbf{t}, H)=\mathbf{t e}-\phi,
$$

we have

$$
\mathbf{e} \delta \mathbf{t}=\delta \psi(\mathbf{t}, H)
$$

at fixed $H$ and

$$
\mathbf{e}=\frac{\partial \psi(\mathbf{t}, H)}{\partial \mathbf{t}}
$$

universally likewise.

2.2. Motivation. In the conventional time-independent formulation of elastic/plastic behavior, a yield surface is assumed at each $H$. This demarcates regions of e- or $\mathbf{t}$-space within which the behavior is elastic and functions $\mathbf{t}(\mathbf{e}, H)$ and $\mathbf{e}(\mathbf{t}, H)$ are defined. The current $\mathbf{t}$ or $\mathbf{e}$ always lies within or on its respective yield surface. In particular, during any program of uninterrupted plastic deformation, e remains on the current yield surface. In that case the functions corresponding 
to each $H$ of the program describe what would have happened if the material had been elastically deformed at that $H$.

By standard thermodynamic arguments applied to states within the elastic domain for any given $H$, one can see that $\phi$ is the free energy. Its additional canonical variable is temperature, but this needs no explicit mention in the present isothermal treatment (cf. Rice [2]). From the standpoint of macroscopic experiment, $\phi$ is defined in each domain via (3) only to within an additive functional of $H$. However, this nonuniqueness is without consequence to our results, which follow from the variation of $\phi$ with $\mathbf{e}$ at a given $H$ or from the difference of this variation at neighboring $H$ 's.

The framework applies as well to the time-dependent formulation of elastic/ plastic behavior, in which the material is imagined to be instantaneously elastic in response to sudden changes in $\mathbf{e}$ or $\mathbf{t}$. Constitutive laws are then such that the inelastic contribution to the rate of deformation is a function of the current stress which is, in general, dependent on the accumulated inelastic history $H$. Hence an instantaneous change in stress causes an instantaneous change in the inelastic contribution to the strain rate, but not to the strain itself. As discussed by Rice [4], this behavior becomes time-independent in the limit of a strongly nonlinear variation of inelastic strain-rate with stress when some history-dependent critical stress range is exceeded. Within such a time-dependent framework, all neighboring strain states are accessible elastically from the current state, at least approximately when the time of strain variation is short in comparison with time scales in the induced plastic flow rate. The elastic response properties to be associated with each state are then those governing this instantaneous elasticity, and $\phi$ is the corresponding free energy.

The formalism which we propose is applicable as well to a broad class of structural models for inelastic behavior, in which the inelasticity is considered to result from microstructural rearrangements of constituent elements of a macroscopic material sample. The extents of these rearrangements are described by a collection of internal state variables; these may, for example, denote the positions of dislocation lines or, at a nonatomistic level of modeling, the pointwise distribution of plastic shears on the different permissible slip systems in each grain of a polycrystal. Here one takes the viewpoint that an energy density may in principle be ascribed to every collection of internal variables and strain (and temperature), whether or not achievable as a corresponding set in an actual deformation. Hence stress may be defined by $\partial \phi / \partial \mathbf{e}$ at constant values of the internal variables, and this relation of $\mathbf{t}$ to $\mathbf{e}$ at fixed internal variables defines the elastic response properties of the material. Indeed, within this framework, $H$ is to be interpreted as symbolizing the current collection of values of the internal variables, and variations in state at fixed $H$ mean variations at fixed internal variables. Note that for this class of models, the $H$-dependent portion of $\phi$ has a definite physical interpretation in terms of the contributions to $\phi$ from the self- and interaction-energies of the internal variables. The corresponding inelastic constitutive laws may be either timeindependent or time-dependent, according to the nature of the kinetic relations adopted to describe the evolution of the internal variables.

2.3. Elastic and plastic increments. Variations in state at fixed $H$ have been denoted by $\delta \mathbf{e}, \delta \phi$, etc., and we shall always use the $\delta$-operator in this sense. More 
general variations that may involve a change $d H$ will henceforth be written $d \mathbf{e}, d \phi$, etc. Also $d^{p}$ will denote the "plastic" change in any constitutive function of $\mathbf{e}$ or $\mathbf{t}$ and $H$; this is defined as the change in the function when $H$ is changed to $H+d H$ but $\mathbf{e}$ or $\mathbf{t}$ respectively is assigned the same value. For example,

$$
\begin{aligned}
d^{p} \phi & =\phi(\mathbf{e}, H+d H)-\phi(\mathbf{e}, H), \\
d^{p} \psi & =\psi(\mathbf{t}, H+d H)-\psi(\mathbf{t}, H) .
\end{aligned}
$$

Here e need bear no relation to the strain at or from which the additional inelastic history $d H$ accumulates, but can take any value within the overlap of the domains of definition of $\phi(\mathbf{e}, H+d H)$ and $\phi(\mathbf{e}, H)$. A similar remark applies to $\mathbf{t}$.

Similarly, with regard to the functions $\mathbf{t}(\mathbf{e}, H)$ and $\mathbf{e}(\mathbf{t}, H)$, the plastic parts of the increments of $\mathbf{t}$ and $\mathbf{e}$ are

$$
d^{p} \mathbf{t}=d \mathbf{t}-\frac{\partial \mathbf{t}}{\partial \mathbf{e}} d \mathbf{e}=d \mathbf{t}-\mathscr{L} d \mathbf{e},
$$

$$
d^{p} \mathbf{e}=d \mathbf{e}-\frac{\partial \mathbf{e}}{\partial \mathbf{t}} d \mathbf{t}=d \mathbf{e}-\mathscr{M} d \mathbf{t}
$$

where $\mathscr{L}$ and $\mathscr{M}$ are the fourth-rank tensors of elastic moduli and compliances. Evidently,

$$
d^{p} \mathbf{t}=-\mathscr{L} d^{p} \mathbf{e} \quad \text { and } \quad d^{p} \mathbf{e}=-\mathscr{M} d^{p} \mathbf{t}
$$

since $\mathscr{L}$ and $\mathscr{M}$ are mutual inverses.

Note that, for a time-independent material, $d^{p} \mathbf{e}$ can generally be effected physically only by a finite cycle of stressing : namely, along a path from a considered t within the domain for $H$ to the boundary point at which $d H$ accumulates and then back to $t$ by a path within the subsequent domain for $H+d H$. Exceptionally, when $\mathbf{t}$ is the point of accumulation itself, an infinitesimal cycle of loading followed by unloading is of course possible. For a time-dependent material, on the other hand, $d^{p} \mathbf{e}$ is the difference between the strains when $\mathbf{t}$ is instantaneously applied after inelastic histories $H$ and $H+d H$ respectively.

The plastic variations $d^{p} \phi$ and $d^{p} \psi$ are themseves functions of e or $\mathbf{t}$ respectively in the overlap of domains for fixed $H$ and $H+d H$. Then, by differentiating (8) as it stands,

$$
\begin{gathered}
\frac{\partial}{\partial \mathbf{e}}\left(d^{p} \phi\right)=\mathbf{t}(\mathbf{e}, H+d H)-\mathbf{t}(\mathbf{e}, H), \\
\frac{\partial}{\partial \mathbf{t}}\left(d^{p} \psi\right)=\mathbf{e}(\mathbf{t}, H+d H)-\mathbf{e}(\mathbf{t}, H),
\end{gathered}
$$

which can be written as

$$
d^{p} \mathbf{t}=\frac{\partial}{\partial \mathbf{e}}\left(d^{p} \phi\right), \quad d^{p} \mathbf{e}=\frac{\partial}{\partial \mathbf{t}}\left(d^{p} \psi\right) .
$$

Thus, the plastic variations in the potentials can be viewed as potentials for the plastic variations in the stress and strain. By virtue of

$$
d^{p} \phi=d \phi-\mathbf{t} d \mathbf{e}, \quad d^{p} \psi=d \psi-\mathbf{e} d \mathbf{t},
$$


which are equivalents of (8), the connection

$$
d^{p} \phi+d^{p} \psi=0
$$

follows at once from (5).

As for purely elastic increments of stress and strain, we need merely note that these are related by

$$
\delta \mathbf{t}=\mathscr{L} \delta \mathbf{e}, \quad \delta \mathbf{e}=\mathscr{M} \delta \mathbf{t}
$$

where, from (4) and (7),

$$
\mathscr{L}=\frac{\partial^{2} \phi}{\partial \mathbf{e}^{2}}, \quad \mathscr{M}=\frac{\partial^{2} \psi}{\partial \mathbf{t}^{2}},
$$

with an obvious notation. Consequently, when arranged as $9 \times 9$ matrices, $\mathscr{L}$ and $\mathscr{M}$ have diagonal symmetry.

2.4. Linear and bilinear differential invariants. If $\rho$ is the mass density in the reference state, the work definition of $\mathbf{t}$ implies that

$$
(1 / \rho) \mathbf{t} d \mathbf{e} \text { is invariant }
$$

to the choices of reference state and strain measure (provided, of course, that every de corresponds to the same change in geometry). Similarly,

$$
(1 / \rho) \delta \phi(\mathbf{e}, H) \text { is invariant at any fixed } H \text {. }
$$

We shall be especially concerned with two kinds of independent differentials emanating from any chosen set of values $\mathbf{t}, \mathbf{e}, H$. One involves purely elastic variations $\delta \mathbf{t}, \delta \mathbf{e}$ related by (14) at the given $H$. The other involves a variation $d H$ in inelastic history, for which plastic variations $d^{p} \mathbf{t}$ and $d^{p} \mathbf{e}$ are defined as in (9). We reiterate that $d H$ is not necessarily accumulated at the chosen values of $\mathbf{t}$ and $\mathbf{e}$.

Consider the scalar products $\delta \mathbf{t} d^{p} \mathbf{e}$ and $\delta \mathbf{e} d^{p} \mathbf{t}$. We refer to these as bilinear forms since they are constructed from a pair of independent differentials. When potentials exist, the forms reduce to

$$
\delta \mathbf{t} d^{p} \mathbf{e}=\delta \mathbf{t} \frac{\partial}{\partial \mathbf{t}}\left(d^{p} \psi\right)=\delta\left(d^{p} \psi\right),
$$

$$
\delta \mathbf{e} d^{p} \mathbf{t}=\delta \mathbf{e} \frac{\partial}{\partial \mathbf{e}}\left(d^{p} \phi\right)=\delta\left(d^{p} \phi\right)
$$

by (11). Here, for example, $\delta\left(d^{p} \phi\right)$ is simply the difference between the values of $d^{p} \phi$ at $\mathbf{e}+\delta \mathbf{e}$ and at $\mathbf{e}$, for the same $H$ and $d H$. Because of (13) we now have the following equivalents :

$$
(1 / \rho) \delta\left(d^{p} \phi\right)=-(1 / \rho) \delta\left(d^{p} \psi\right)=(1 / \rho) \delta \mathbf{e} d^{p} \mathbf{t}=-(1 / \rho) \delta \mathbf{t} d^{p} \mathbf{e} .
$$

But $\delta\left(d^{p} \phi\right) \equiv d^{p}(\delta \phi)$, where the latter signifies the difference of $\delta \phi$ values at $H$ and $H+d H$ for the same neighboring geometries (since $\phi$ is a function of $\mathbf{e}$ ). Consequently $d^{p}(\delta \phi) / \rho$ is an invariant by (17). It can therefore be concluded that each member of (19) is invariant to choices of strain measure and reference state.

Substitution of formulas (9) into the respective members of (19) yields

$$
(1 / \rho) \delta \mathbf{e}(d \mathbf{t}-\mathscr{L} d \mathbf{e}) \quad \text { and } \quad-(1 / \rho) \delta \mathbf{t}(d \mathbf{e}-\mathscr{M} d \mathbf{t}) .
$$


Since $\mathscr{L}$ and $\mathscr{M}$ are here symmetric, these are the same as

$$
(1 / \rho)(d \mathbf{t} \delta \mathbf{e}-\delta \mathbf{t} d \mathbf{e}) .
$$

Thus (19) is but a special case of Hill's [3] bilinear invariant

$$
(1 / \rho)\left(d_{1} \mathbf{t} d_{2} \mathbf{e}-d_{2} \mathbf{t} d_{1} \mathbf{e}\right),
$$

where $d_{1}$ and $d_{2}$ are independent differentials and no material properties are involved.

By integrating each member of (19) between any two states $A$ and $B$ within an $H$-domain, considering $d H$ fixed, one obtains

$$
\frac{1}{\rho}\left[d^{p} \phi\right]_{A}^{B}=-\frac{1}{\rho}\left[d^{p} \psi\right]_{A}^{B}=\frac{1}{\rho} \int_{A}^{B} d^{p} \mathbf{t} \delta \mathbf{e}=-\frac{1}{\rho} \int_{A}^{B} d^{p} \mathbf{e} \delta \mathbf{t} .
$$

These may be regarded as further linear invariants.

3. Normality in conjugate variables. Here we examine some postulates that lead to "normality rules" in conjugate stress and strain variables for inelastic solids. By means of the invariants established previously, it will be shown that such rules apply for every choice of reference state and strain measure, or for none. We shall subsequently study their transmissibility to conjugate macrovariables for composite continua. Finally, we give a specification of incremental or rate constitutive laws for internal variable models which are sufficient to ensure normality.

3.1. Normality rules for time-independent plasticity. In the time-independent idealization of elastic/plastic behavior, a yield surface is assumed to exist in t- (or e-) space at each history $H$. Let $d^{p} \mathbf{e}$ be the plastic variation in strain associated with the stress $\mathbf{t}$ under which $d H$ occurs; then $d^{p} \mathbf{e}$ is the residual strain-increment in an infinitesimal load-unload cycle. Further, let $\delta \mathbf{t}$ be any stress increment emanating from the same $\mathbf{t}$ and directed inside the yield surface. If

$$
\delta \mathbf{t} d^{p} \mathbf{e}<0
$$

for every such $\delta \mathbf{t}$, we shall say that the material complies with the normality rule. Well-known consequences are that $d^{p} \mathbf{e}$ must be codirectional with the outward normal to a locally smooth yield surface in $\mathbf{t}$-space, whereas at a vertex it must lie within or on the cone of limiting outward normals.

From (19) it is evident that (22) implies

$$
\delta \mathbf{e} d^{p} \mathbf{t}>0 .
$$

This expresses a dual normality requiring $d^{p} \mathbf{t}$ to be codirectional with the inward normal to a yield surface in e-space, with a similar generalization at a vertex.

From (18) or (19), each of these inequalities is equivalent to either of

$$
\delta\left(d^{p} \psi\right)<0, \quad \delta\left(d^{p} \phi\right)>0 .
$$

Hence the normality rule amounts to the following local minimum property: $d^{p} \phi$ when evaluated at the state of flow is less than $d^{p} \phi$ when evaluated at any immediately neighboring state within the yield surface. Since all members of (19) were proved to be invariants, each normality inequality applies to all conjugate measures or to none. 
3.2. Cycle inequalities for time-independent materials. The Il'yushin [5] postulate is

$$
\oint \mathbf{t} d \mathbf{e} \geqq 0,
$$

where the integral is taken around a strain cycle, and the strict inequality holds when inelastic deformation occurs at some stage. Being based on work, the postulate is automatically invariant to reference state and measure. Further, within the present context of finite strain, Hill [6] has shown that the normality rule (22) in conjugate variables results from the postulate when applied to certain limiting types of infinitesimal cycles.

Here we consider the inequality for finite cycles, but only ones that involve a first order inelastic history $d H$. This is accumulated at a state $\mathbf{e}_{*}$, say, on a yield surface, whereas the path starts from some finitely distant state $\mathbf{e}_{0}$ within the surface. A cycle consists of three segments : (a) $\mathbf{e}_{0} \rightarrow \mathbf{e}_{*}$ elastically at history $H$; (b) $\mathbf{e}_{*} \rightarrow \mathbf{e}_{*}$ $+d \mathbf{e}_{*}$ with an accumulation of history $d H$, followed by $\mathbf{e}_{*}+d \mathbf{e}_{*} \rightarrow \mathbf{e}_{*}$ elastically at $H+d H$; (c) $\mathbf{e}_{*} \rightarrow \mathbf{e}_{0}$ elastically at $H+d H$, which is always possible for sufficiently small $d H$.

Segment (b) contributes only a term of second order to the integral. Also, because of path-independence within an elastic domain, segment (c) can be chosen to retrace (a). It is then apparent that, to first order,

$$
\oint \mathbf{t} d \mathbf{e}=\int_{\mathbf{e}_{0}}^{\mathbf{e}_{*}}\{\mathbf{t}(\mathbf{e}, H)-\mathbf{t}(\mathbf{e}, H+d H)\} \delta \mathbf{e}=-\int_{\mathbf{e}_{0}}^{\mathbf{e}_{*}} d^{p} \mathbf{t} \delta \mathbf{e},
$$

where the $\delta$-notation signifies as usual that $H$ and $d H$ are fixed during the integration (so that $d^{p} \mathbf{t}$ varies only with $\mathbf{e}$ ). Thus, by (11),

$$
\oint \mathbf{t} d \mathbf{e}=\left[d^{p} \phi\right]_{\mathbf{e}_{*}}^{\mathbf{e}_{*}}
$$

This incidentally supplies a work interpretation of the invariants in (19) and of the potentials in (11) and (13).

Our restricted version of the Il'yushin postulate thus leads to global forms of (24):

$$
\left[d^{p} \psi\right]_{\mathbf{t}_{*}}^{\mathrm{t}_{0}}<0, \quad\left[d^{p} \phi\right]_{\mathbf{e}_{*}}^{\mathbf{e}_{0}}>0
$$

It is recalled that $\left(\mathbf{t}_{0}, \mathbf{e}_{0}\right)$ is any state inside an $H$-surface, while $\left(\mathbf{t}_{*}, \mathbf{e}_{*}\right)$ is the yieldpoint state for $d H$.

3.3. Flow potential for time-dependent plasticity. Consider time-dependent materials exhibiting instantaneous elasticity. For the plastic portion of the rate of strain, we assume the constitutive relation

$$
\frac{d \mathbf{e}}{d \theta}-\mathscr{M} \frac{d \mathbf{t}}{d \theta}=\frac{d^{p} \mathbf{e}}{d \theta}(\mathbf{t}, H)
$$

where $\theta$ denotes time and the right-hand side is a function of the current stress and a functional of inelastic history. 
Since instantaneous variations of stress induce only an elastic instantaneous response, it is meaningful to discuss the functional dependence of $d^{p} \mathbf{e} / d \theta$ on $\mathbf{t}$ at any fixed $H$. If this is such that $\delta \mathbf{t}\left(d^{p} \mathbf{e} / d \theta\right)$ is a perfect differential at fixed $H$, then a scalar "flow potential" function of $\mathbf{t}$ exists at each $H$ so that

$$
\delta \mathbf{t} \frac{d^{p} \mathbf{e}}{d \theta}=\delta \Omega(\mathbf{t}, H)
$$

that is,

$$
\frac{d^{p} \mathbf{e}}{d \theta}=\frac{\partial \Omega(\mathbf{t}, H)}{\partial \mathbf{t}}
$$

Since multiplication by $d \theta / \rho$ converts (29) into one of the bilinear invariants (19), the normality rule for the flow potential is also invariant to the choices of strain measure and reference state. Further, since

$$
\delta \mathbf{t} \frac{d^{p} \mathbf{e}}{d \theta}=-\delta \mathbf{e} \frac{d^{p} \mathbf{t}}{d \theta}
$$

we also have

$$
\frac{d^{p} \mathbf{t}}{d \theta}=-\frac{\partial \Omega(\mathbf{e}, H)}{\partial \mathbf{e}}
$$

when $\Omega$ is expressed in terms of $\mathbf{e}$ and $H$.

Rice [4], [2] has commented on the manner in which the normality rule in time-independent plasticity might be considered as a singular limit of the rate equations (30) and (31).

4. Heterogeneous aggregates. We consider heterogeneous media such as polycrystals or fiber composites or, for that matter, structural systems, and treat them as aggregates of continuous subelements in each of which the $\mathbf{t}$ - and $\mathbf{e}$-fields comply with the constitutive framework in $\S 2$. A representative volume of the aggregate is subjected to surface loadings of a type for which conjugate generalized forces and displacements are definable; we denote these respectively by $Q_{i}, q_{i}$, $i=1,2, \cdots, n$, or collectively by $Q, q$ in state functions. Hill [7], [3] and Rice [2] have discussed how they can be set in correspondence with macroscopic conjugate stresses and strains, when these are defined appropriately for the aggregate. Our initial aim is to express plastic variations of the $q$ 's in terms of local $d^{p} \mathbf{e}$ values within the subelements. Subsequently, we show how normality rules assumed for the subelement materials are necessarily transmitted to the macroscopic constitutive laws.

4.1. Potentials for the aggregate. Suppose the aggregate exhibits elastic behavior such that the $Q$ 's are determined by the $q$ 's together with the inelastic history $H$. By the virtual work principle, and with the summation convention,

$$
Q_{i} d q_{i}=\int(\mathbf{t} d \mathbf{e}) d V,
$$

where the integral is taken over the volume $V$ of the aggregate in a reference configuration relative to which each local $\mathbf{e}$ is reckoned. 
In particular, when local potentials $\phi(\mathbf{e}, H)$ exist and every subelement behaves elastically, $Q_{i} \delta q_{i}=\delta \Phi$, where

$$
\Phi(q, H)=\int \phi(\mathbf{e}, H) d V
$$

In these circumstances $\Phi$ acts as a macropotential, in that

$$
Q_{i}=\partial \Phi / \partial q_{i} .
$$

Dually, when the $Q$ 's and $H$ are the independent variables,

$$
q_{i}=\partial \Psi / \partial Q_{i},
$$

where

$$
\Psi(Q, H) \equiv Q_{i} q_{i}-\Phi(q, H)
$$

by the Legendre transformation. However, $\Psi$ is generally not the volume integral of $\psi$. Exceptionally, for infinitesimal strains and under the usual linearizations,

$$
Q_{i} q_{i}=\int \text { te } d V \text { and } \Psi(Q, H)=\int \psi(\mathbf{t}, H) d V
$$

in the reference configuration.

As in $\S 2.3$, plastic variations can be defined for the macropotentials and the generalized variables; for example,

$$
\begin{aligned}
& d^{p} \Phi=\Phi(q, H+d H)-\Phi(q, H), \\
& d^{p} q_{i}=q_{i}(Q, H+d H)-q_{i}(Q, H) .
\end{aligned}
$$

Then, by following the procedure leading to (11),

$$
d^{p} Q_{i}=\frac{\partial}{\partial q_{i}}\left(d^{p} \Phi\right), \quad d^{p} q_{i}=\frac{\partial}{\partial Q_{i}}\left(d^{p} \Psi\right)
$$

where

$$
d^{p} \Phi+d^{p} \Psi=0 .
$$

Now consider aggregate states $(q, H)$ and $(q, H+d H)$, where the increment $d H$ in inelastic history may accumulate at other generalized displacements. Let $\mathbf{e}$ and $\mathbf{e}+d \mathbf{e}$ be the local strains corresponding to $(q, H)$ and $(q, H+d H)$ respectively. Of course, returning the $q$ 's to their pre- $d H$ values does not generally restore the local strains, and in that event de is a nonzero field. Nevertheless, since it corresponds to zero $d q$ 's,

$$
\int \mathbf{t} d \mathbf{e} d V=0
$$

by virtual work, where $\mathbf{t}$ is the stress field for the state $(q, H)$. Now

$$
\begin{aligned}
\Phi(q, H+d H)-\Phi(q, H) & =\int\{\phi(\mathbf{e}+d \mathbf{e}, H+d H)-\phi(\mathbf{e}, H)\} d V \\
& =\int\left(\mathbf{t} d \mathbf{e}+d^{p} \phi\right) d V
\end{aligned}
$$


as in (12) and so, by (37),

$$
d^{p} \Phi=\int d^{p} \phi d V
$$

Further, from (13) and (36),

$$
d^{p} \Psi=\int d^{p} \psi d V
$$

even though $\Psi$ itself is not the volume integral of $\psi$.

We have thereby proved that in a cycle of the $q$ 's ( $Q^{\prime}$ 's) the plastic part $d^{p} \Phi\left(d^{p} \Psi\right)$ is the volume integral of $d^{p} \phi\left(d^{p} \psi\right)$, regardless of whether the local variables are simultaneously cycled.

4.2. Plastic increments of generalized force and displacement. The connections (38) and (39) apply in any state within the overlap of elastic domains for the aggregate after histories $H$ and $H+d H$. Hence taking $\delta$ variations in this overlap,

$$
\delta\left(d^{p} \Phi\right)=\int \delta\left(d^{p} \phi\right) d V, \quad \delta\left(d^{p} \Psi\right)=\int \delta\left(d^{p} \psi\right) d V .
$$

Consequently, by (36),

$$
\delta q_{i} d^{p} Q_{i}=\int\left(\delta \mathbf{e} d^{p} \mathbf{t}\right) d V, \quad \delta Q_{i} d^{p} q_{i}=\int\left(\delta \mathbf{t} d^{p} \mathbf{e}\right) d V,
$$

since (11) holds at every point of the aggregate. Thus the bilinear invariants in the generalized variables are just volume integrals of the corresponding micro-invariants.

Equivalent to (41) are

$$
d^{p} Q_{i}=\int\left(\frac{\partial \mathbf{e}}{\partial q_{i}} d^{p} \mathbf{t}\right) d V, \quad d^{p} q_{i}=\int\left(\frac{\partial \mathbf{t}}{\partial Q_{i}} d^{p} \mathbf{e}\right) d V
$$

These are explicit expressions for the plastic parts of increments in the generalized variables. In the partial derivatives $H$ is kept fixed, the two "influence tensor functions" being well-defined in principle as solutions of elastic boundary value problems for the aggregate. This standpoint has been developed systematically by Hill [8] in the linear case.

These results could be derived alternatively as special cases of Hill's [3] general theorem that the integral of the bilinear invariant (20) over a representative volume is equal to the same invariant in the macroscopic variables. That is, in the present notation,

$$
\left.\begin{array}{l}
d\left(Q_{i} \delta q_{i}\right)-\delta\left(Q_{i} d q_{i}\right) \\
\delta\left(q_{i} d Q_{i}\right)-d\left(q_{i} \delta Q_{i}\right)
\end{array}\right\}=\left\{\begin{array}{l}
\int\{d(\mathbf{t} \delta \mathbf{e})-\delta(\mathbf{t} d \mathbf{e})\} d V \\
\int\{\delta(\mathbf{e} d \mathbf{t})-d(\mathbf{e} \delta \mathbf{t})\} d V .
\end{array}\right.
$$

When potentials exist, the integrands in (41) and (43) are related in the way detailed in $\S 2.4$. Correspondingly, the left-hand side of (43) reduces to either of

and in (40).

$$
\begin{aligned}
d(\delta \Phi)-\delta\left(d \Phi-d^{p} \Phi\right) & =\delta\left(d^{p} \Phi\right), \\
\delta\left(d \Psi-d^{p} \Psi\right)-d(\delta \Psi) & =-\delta\left(d^{p} \Psi\right),
\end{aligned}
$$


4.3. Transmission of normality rules. Each normality rule in $\S 3$ is now easily seen to hold for the aggregate if it is asserted for all subelement materials.

Taking first the time-independent case, it follows from (40) that the equivalent inequalities (22) and (23) imply that

$$
\delta Q_{i} d^{p} q_{i}<0, \quad \delta q_{i} d^{p} Q_{i}>0 .
$$

These can be stated as normality rules for yield surfaces in the $Q$ - and $q$-spaces. Likewise, variational versions of the rule follow from (24) and (40):

$$
\delta\left(d^{p} \Psi\right)<0, \quad \delta\left(d^{p} \Phi\right)>0 .
$$

The above inequalities apply when some part of the aggregate is currently at yield and a $\delta$-increment causes that part to unload.

Suppose, next, that the aggregate is taken through a cycle of the $q$ 's, consisting of $q_{0} \rightarrow q_{*}$ elastically at history $H, q_{*} \rightarrow q_{*}+d q_{*}$ inelastically, $q_{*}+d q_{*} \rightarrow q_{0}$ elastically at history $H+d H$. Then, by steps analogous to those leading to (26),

$$
\oint Q_{i} d q_{i}=\left[d^{p} \Phi\right]_{q_{*}^{*}}^{q_{0}}
$$

But, by (38), even though the e-field may not be restored in the cycle, (46) can be put as

$$
\oint Q_{i} d q_{i}=\int\left[d^{p} \phi\right]_{\mathbf{e}_{*}}^{\mathbf{e}_{0}} d V
$$

where the integral extends only over those parts of the aggregate that contribute to $d H$. If, now, (27) holds for all subelement materials, then (47) implies that

$$
\oint Q_{i} d q_{i}>0
$$

for $q$-cycles of the specified kind. Thus the restricted Il'yushin postulate is transmitted to the generalized variables.

It is perhaps worth remarking that, at either level, the restricted postulate implies an expenditure of work in certain cycles with finite $H$ accumulation, namely, via states from which the initial configuration can be regained elastically. For, clearly, any such path is both geometrically and energetically equivalent to a simple sum of infinitesimal cycles of the above kind.

For time-dependent materials with instantaneous elasticity, the rate version of $(42)$ is

$$
\frac{d^{p} q_{i}}{d \theta}=\int\left\{\frac{\partial \mathbf{t}(Q, H)}{\partial Q_{i}} \frac{d^{p} \mathbf{e}}{d \theta}\right\} d V,
$$

where the notation emphasizes that the t-fleld is in principle determined by the $Q$ 's at any $H$. Hence, if a flow potential exists for the material of every subelement, so that (30) applies, then

$$
\begin{aligned}
\frac{d^{p} q_{i}}{d \theta} & =\int\left\{\frac{\partial \mathbf{t}(Q, H)}{\partial Q_{i}} \frac{\partial \Omega(\mathbf{t}, H)}{\partial \mathbf{t}}\right\} d V \\
& =\frac{\partial}{\partial Q_{i}} \int \Omega(\mathbf{t}, H) d V .
\end{aligned}
$$


This shows that a flow potential exists for the aggregate and is just the volume integral of the local flow potentials.

\section{Internal variables.}

5.1. Internal variable representations of $H$. Our approach is motivated by earlier internal variable formulations of inelasticity, and here we draw the connection between the two. Rice $[2, \S 2.6]$ has distinguished between internal variables of the "specific structural" type and "averaging" type. The former are introduced within a framework paralleling that of the last section, in which a single, representative, macroscopic sample of material is considered. The variables characterize specific structural rearrangements (e.g., crystalline slip) taking place at individual sites within the sample. The required number of variables is thereby proportional to the mass of the sample, since the number of operative sites increases proportionally. By contrast, the latter type of variable refers to averaged characterizations of the rearrangements taken over all the operative sites. We may consider the replacement of the essentially infinite number of specific structural variables by averaging variables to have been accomplished a priori, or instead to be later accomplished once the complete constitutive framework is formulated in terms of the specific variables. In either event, the development of meaningful averages may be considered as a separate problem from that addressed here, and the following discussion applies to a formulation based on either type of internal variable.

Referring to $\S 2.2$, behavior at fixed $H$ is now understood to mean behavior at a fixed set of internal variables; $d H$ is understood to correspond to increments $d \xi_{1}, d \xi_{2}, \cdots, d \xi_{n}$ of a possibly infinite number of internal variables which are, in general, nonholonomic. Hence if energetic "forces" $F_{i}=F_{i}(\mathbf{e}, H)$ are defined as the negative change in $\phi$ induced per unit $d \xi_{i}$, at fixed $\mathbf{e}$ and other internal variables, then

$$
d^{p} \phi=-F_{i}(\mathbf{e}, H) d \xi_{i}
$$

with the summation convention. Dually, by (13),

$$
d^{p} \psi=F_{i}(\mathbf{t}, H) d \xi_{i},
$$

where now the $F$ 's are expressed in terms of $\mathbf{t}$. (In the case of specific structural variables, $F_{i}$ is more conveniently written as a conjugate force $f_{i}$ divided by the reference state volume of the sample: $F_{i}=f_{i} / V$.)

From (11) applied to the above representations of $d^{p} \phi, d^{p} \psi$, we have

$$
d^{p} \mathbf{t}=-\frac{\partial F_{i}(\mathbf{e}, H)}{\partial \mathbf{e}} d \xi_{i}, \quad d^{p} \mathbf{e}=\frac{\partial F_{i}(\mathbf{t}, H)}{\partial \mathbf{t}} d \xi_{i} .
$$

These are Rice's [2] equations relating increments of the internal variables to plastic portions of stress and strain increments.

Alternatively, it is possible to begin the formulation with equations of the kind

$$
d^{p} \mathbf{e}=\boldsymbol{\mu}_{i}(\mathbf{t}, H) d \xi_{i},
$$

where $\boldsymbol{\mu}_{i}$ is the $d^{p} \mathbf{e}$ induced per unit $d \xi_{i}$. Then, in view of (11),

$$
d^{p} \psi=\int^{\mathbf{t}} d^{p} \mathbf{e} \delta \mathbf{t}=d \xi_{i} \int^{\mathbf{t}} \boldsymbol{\mu}_{i}(\mathbf{t}, H) \delta \mathbf{t},
$$


where the integration is on t at fixed $H$ and $d H$ (or $d \xi^{\prime}$ s). But $d^{p} \psi$ is a direct function of $\mathbf{t}$, and this must be the case for any combination of $d \xi$ 's. Hence, the integral of each $\boldsymbol{\mu}_{i}$ is path-independent, and thus potentials which we shall denote by $F_{i}(\mathbf{t}, H)$ must exist so that

$$
\boldsymbol{\mu}_{i}=\frac{\partial F_{i}(\mathbf{t}, H)}{\partial \mathbf{t}} .
$$

It is then evident that (55) leads to an expression of the form (52) for $d^{p} \psi$. A similar result could, of course have been obtained beginning with an equation analogous to (54) for $d^{p}$ t. Rice [2] and Hill and Rice [9] have shown the physical identification of the $F$ 's and $\mu$ 's in the case of crystalline slip models for metal plasticity.

5.2. Differential invariants and normality rules. The families (19) and (21) of invariants have representations in terms of the internal variables and their conjugate forces. Starting from any of (51), (52), or (53), the family (19) includes

$$
\frac{1}{\rho} \delta\left(d^{p} \phi\right)=\cdots=-\frac{1}{\rho} \delta \mathbf{t} d^{p} \mathbf{e}=-\frac{1}{\rho} \delta F_{i} d \xi_{i}
$$

and the family (21) includes

$$
\frac{1}{\rho}\left[d^{p} \phi\right]_{A}^{B}=\cdots=-\frac{1}{\rho} \int_{A}^{B} d^{p} \mathbf{e} \delta \mathbf{t}=-\frac{1}{\rho}\left(F_{i}^{B}-F_{i}^{A}\right) d \xi_{i} .
$$

From these it is clear that the validity of the normality rules of $\S 3$ will hinge on properties of the constitutive laws for evolution of the internal variables. Thermodynamics restricts the relations only by $F_{i} d \xi_{i} / d \theta \geqq 0$. The simplest assumption, which reduces, as Rice [2] has shown, to conventional notions concerning the stress dependence of crystalline slip, is that at any given $H$, the rate of change $d \xi_{i} / d \theta$ is a function of $\mathbf{t}$ or $\mathbf{e}$ only via the associated force $F_{i}$. The corresponding statement in the time-independent case is that the yield criterion for the ith variable is phrased solely in terms of $F_{i}: F_{i}^{L}<F_{i}<F_{i}^{U}$, where the limits $F_{i}^{L}, F_{i}^{U}$ depend on $H$ and yield emanating from $F_{i}^{L}$ involves $d \xi_{i}<0$, whereas that emanating from $F_{i}^{U}$ involves $d \xi_{i}>0$.

For such constitutive laws, it is evident from the last version of (57) that each of the normality inequalities of $\S 3.1$ for the time-dependent case is valid because, for example,

$$
\delta \mathbf{t} d^{p} \mathbf{e}=\delta F_{i} d \xi_{i}<0
$$

where the inequality applies since it is trivially true for each separate bilinear term in the sun. Also, by using the last version of (58) and (26), the Il'yushin integral of $\S 3.2$ becomes

$$
\oint \mathbf{t} d \mathbf{e}=\left(F_{i}^{*}-F_{i}^{0}\right) d \xi_{i}^{*}>0
$$

where again the inequality applies because it is true for each separate term in the sum. 
In the time-dependent case of $\S 3.3$, we may write

$$
\delta \mathbf{t} \frac{d^{p} \mathbf{e}}{d \theta}=\delta F_{i} \frac{d \xi_{i}}{d \theta}
$$

from (57). Now, for the class of constitutive laws under consideration, each term of the sum on the right is a perfect differential because each $d \xi_{i} / d \theta$ depends only on the associated $F_{i}$ and $H$. Hence the term on the left is also a perfect differential and this means that a flow potential exists as in (29) and (30).

\section{REFERENCES}

[1] J. Kestin And J. R. Rice, Paradoxes in the application of thermodynamics to strained solids, A Critical Review of Thermodynamics, E. B. Stuart et al., eds., Mono Book Corp., Baltimore, 1970, pp. 275-298.

[2] J. R. RICE, Inelastic constitutive relations for solids: an internal variable theory and its application to metal plasticity, J. Mech. Phys. Solids, 19 (1971), pp. 433-455.

[3] R. Hill, On constitutive macro-variables for heterogeneous solids at finite strain, Proc. Roy. Soc. London Ser A, 326 (1972), pp. 131-147

[4] J. R. RICE, On the structure of stress-strain relations for time-dependent plastic deformation in metals, Trans. ASME Ser. E J. Appl. Mech., 37 (1970), pp. 728-737.

[5] A. A. IL'yushin, On a postulate of plasticity, Prikl. Mat. Meh., 25 (1961), pp. 503-507.

[6] R. HiLl, On constitutive inequalities for simple materials-II, J. Mech. Phys. Solids, 16 (1968), pp. 315-322.

[7] - The essential structure of constitutive laws for metal composites and polycrystals, Ibid., 15 (1967), pp. 79-95.

[8] - On macroscopic measures of plastic work and deformation in micro-heterogeneous media, Prikl. Met. Meh., 35 (1971), pp. 31-39.

[9] R. Hill AND J. R. Rice, Constitutive analysis of elastic/plastic crystals at arbitrary strain, J. Mech. Phys. Solids, 20 (1972), pp. 401-413. 